\title{
Perturbações músculo-esqueléticas em adolescentes: estudo da prevalência e dos fatores determinantes
}

\section{Musculoskeletal disorders in adolescents: a study on prevalence and determining factors} Trastornos musculoesqueléticos en adolescentes: estudio de prevalencia y factores determinantes

Rosa Lopes Martins ib https://orcid.org/0000-0001-9850-9822.

Nélia Carvalho it https://orid.org/0000-0002-7065-5366

Carlos Albuquerque io https://orcid.org/0000-0002-2297-0636'

Ana Andrade ic https://orcid.org/0000-0003-1070-8507!

Conceição Martins ị https://orcid.org/0000-0002-6598-822x

Sofia Campos io hitps://orcid.org/0000-0002-4696-3537'

Susana Batista io https://orcid.org/0000-0003-0256-6027'

Alexandra Isabel Dinis io hitps://orcid.org/0000-0002-4102-53221

\section{Como citar:}

Martins RL, Carvalho N, Albuquerque C, Andrade A, Martins C, Campos S, et al.

Perturbações músculo-esqueléticas em

adolescentes: estudo da prevalência e dos

fatores determinantes. Acta Paul Enferm. 2020;33:e-APE20190173

DOI

http://dx.doi.org/10.37689/ acta-ape/2020A00173

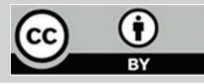

Adolescente; Dor músculoesquelética/ epidemiologia; Prevalência; Saúde escolar

Keyowords

Adolescent; Musculoskeletal pain/epidemiology; Prevalence; School health

Descriptores

Adolescente; Dolor musculoesquelético/ epidemiología; Prevalencia; Salud escolar

Submetido

15 de Julho de 2019

Aceito

22 de Julho de 2019

\section{Autor correspondente}

Rosa Lopes Martins

https://orcid.org/0000-0001-9850-9822

E-mail: rmartins.viseu@gmail.com

\section{Resumo}

Objetivo: Identificar a prevalência das perturbações músculo-esqueléticas em adolescentes e analisar a sua relação com variáveis sociodemográficas, antropométricas e contextuais.

Métodos: Conceptualizou-se um estudo observacional, transversal, descritivo/relacional de cariz quantitativo, que envolveu 632 adolescentes de seis agrupamentos de escolas das zonas centro e norte de Portugal, com média de idades de 13,36 anos. 0 protocolo de colheita de dados integrava questões de cariz sociodemográfico, antropométrico, contextual e ainda o "Questionário Nórdico Músculo-Esquelético" para avaliar as perturbações músculo-esqueléticas.

Resultados: Os dados mostraram que 47,4\% dos adolescentes referem perturbação músculo-esqueléticas nos últimos 3 meses, localizando-se estas aos níveis dos ombros (27,8\%), região dorsal (25,3\%), coxa/anca $(26,1 \%)$, pescoço $(23,4 \%)$, zona lombar $(22,8 \%)$ e joelhos $(19,6 \%)$. Verificou-se ainda, que as perturbações músculo-esqueléticas são mais prevalentes nos adolescentes do género feminino, naqueles que gastam mais tempo por dia a ver televisão e ainda nos que se deslocam para a escola a pé ou de bicicleta.

Conclusão: A prevalência das perturbações músculo-esqueléticas nos adolescentes é elevada, tem origem multicausal, tornando-se imperativa a implementação de intervenções de prevenção e readaptação promotoras de um funcionamento músculo-esquelético otimizado.

\section{Abstract}

Objective: To identify the prevalence of musculoskeletal disorders in adolescents, and to analyze this prevalence relationship with sociodemographic, anthropometric and contextual variables.

Methods: An observational, cross-sectional, descriptive/relational study with quantitative approach was conceptualized, involving 632 adolescents with mean age of 13.36 from six school groups from Central and North Portugal. Data collection protocol included questions of sociodemographic, anthropometric and contextual nature, and also the "Nordic Musculoskeletal Questionnaire" to assess musculoskeletal disorders.

Results: Data showed that $47.4 \%$ of adolescents mentioned musculoskeletal disorders within the last 3 months, located in the shoulders (27.8\%), dorsal region (25.3\%), thigh/hips (26.1\%), neck (23.4\%), lumbar region (22.8\%), and knees (19.6\%). We found that musculoskeletal disorders are more prevalent in female adolescents, and among those who spend longer time watching television per day, and also those who go to school by foot or bicycle.

Conclusion: The prevalence of musculoskeletal disorders in adolescents is high and has multicausal origin, turning imperative the implementation of preventive and readapted actions to promote a better musculoskeletal function. 


\section{Resumen}

Objetivos: Identificar la prevalencia de trastornos musculoesqueléticos en adolescentes y analizar su relación con variables sociodemográficas, antropométricas y contextuales.

Métodos: Se conceptualizó un estudio observacional, transversal, descriptivo/relacional de aspecto cuantitativo, que incluyó 632 adolescentes de seis agrupamientos de escuelas de la zona centro y norte de Portugal, con promedio de edad de 13,36 años. El protocolo de recolección de datos incluyó cuestiones de aspecto sociodemográfico, antropométrico, contextual e, inclusive, el "Cuestionario Nórdico Musculoesquelético" para evaluar los trastornos musculoesqueléticos.

Resultados: Los datos mostraron que el 47,4\% de los adolescentes señalan trastornos musculoesqueléticos en los últimos 3 meses, localizados en el nivel de los hombros (27,8\%), región dorsal (25,3\%), muslo/cadera $(26,1 \%)$, cuello $(23,4 \%)$, zona lumbar $(22,8 \%)$ y rodillas (19,6\%). Además, se verificó que los trastornos musculoesqueléticos tienen mayor prevalencia en adolescentes de género femenino, en los que pasan más tiempo por día viendo televisión y en Ios que van a la escuela caminando 0 en bicicleta.

Conclusión La prevalencia de los trastornos musculoesqueléticos en adolescentes es elevada, tiene origen multicausal, por lo que se torna imperativa la implementación de intervenciones de prevención y readaptación que promuevan un funcionamiento musculoesquelético optimizado.

\section{Introdução}

A Organização Mundial de Saúde (OMS) ${ }^{(1)}$ conceptualiza adolescencia como a fase de vida compreendida entre os 10 e 19 anos, tratando-se de um período de transição entre a infância e a idade adulta. Caracteriza-se pelo desenvolvimento físico, mental e emocional, onde são feitos esforços no sentido de serem alcançadas diferentes metas, de acordo com a cultura de cada um. É uma fase de vida que se inicia com as mudanças corporais da puberdade e termina quando o indivíduo consolida o seu crescimento e personalidade, obtendo progressivamente independência económica e integração no grupo social. ${ }^{(2)}$

Trata-se portanto de um periodo marcado por intensas modificaçóes físicas, psíquicas, comportamentais e sociais, constituindo a transição entre a infância e a vida adulta, em que muitas das características ou dos hábitos referentes ao estilo de vida do adulto são adquiridos e/ou consolidados. Também é neste periodo temporal, que o ser humano apresenta aceleração na velocidade de crescimento da estatura e no ganho de peso, o que justifica o aumento das necessidades nutricionais. ${ }^{(3)}$

Os estudos biomecânicos do corpo humano têm mostrado, que há comportamentos posturais que causam desequilíbrios no sistema corporal, levando a compensaçóes que podem provocar alteraçóes em suas estruturas e funções. Sabe-se que durante a puberdade, se verifica assimetria no crescimento, uma vez que os ossos crescem mais rapidamente que os músculos e tendôes, sendo este facto notório sobretudo a nivel da coluna vertebral. $\mathrm{O}$ adolescente leva algum tempo a adaptar-se ao seu novo corpo numa fase em que é frequente a introspeção, sensibilidade e até vergonha do corpo e por isso ser comum uma postura encolhida, que propicia o aparecimento de lesôes. ${ }^{(4)}$

A perturbação músculo-esquelética (PME) do adolescente concetualiza-se como presença de mau estar, dor ou desconforto. ${ }^{(5)}$ Trata-se de uma entidade nosológica que afeta músculos, nervos, discos intervertebrais, articulações, cartilagens, tendões e ligamentos, manifestando-se de forma pontual, sistemática ou crónica. A sua etiologia pode ser genética ou adquirida, porém condiciona de forma mais ou menos significativa as tarefas de vida diária e a qualidade de vida dos individuos. ${ }^{(6)}$

Estudos epidemiológicos realizados em adolescentes, sugerem que a prevalência e a incidência têm aumentado substancialmente nos últimos anos, incluindo Portugal, podendo mesmo evoluir para situaçôes de cronicidade que acompanham a vida adulta. ${ }^{(7)}$ Dos múltiplos estudos levados a cabo pelos diferentes investigadores tem emergido o consenso de que as PME não podem ser apenas explicados por factores físicos, mas sim por factores sociais, psicológicos, ambientais e comportamentais que precipitam as manifestaçóes deste problema. ${ }^{(2,3,8,9)}$

Assim, face à complexidade descrita e á dimensão crescente do problema, investigar a prevalência destas perturbaçóes na infância e adolescência, reconhecer os fatores que contribuem para o seu aparecimento e avaliar o impacto de medidas de promoção da qualidade de vida, constituem hoje um grande desafio não só para os profissionais de saúde, mas para a população em geral. De facto, o impacto negativo que estas perturbaçóes causam não só nesta fase de vida mas também na vida adulta, constituem hoje em dia um verdadeiro problema de saúde pública. ${ }^{(5,8)}$ 
Emerge assim a necessidade de melhorar as estratégias de prevenção individuais, biomecânicas, organizacionais e psicossociais instituídas na prevenção de $\mathrm{PME}$, onde a intervenção dos profissionais de saúde, em articulação com equipas muldisciplinares, deve ser potencialmente promovida e implementada.

Considerando os pressupostos descritos e a dimensão do problema, desenvolvemos esta pesquisa com o objetivo de identificar perturbaçôes músculo -esqueléticas em adolescentes e determinar relações existentes, entre as PME e variáveis de carácter sociodemográfico, antropométrico e contextual.

\section{Métodos}

Conceptualizou-se um estudo observacional, transversal, descritivo/relacional de cariz quantitativo. A amostra utilizada é do tipo não probabilístico por conveniência, constituída por 632 adolescentes de seis Agrupamentos de Escolas das zonas centro e norte de Portugal. A elegibilidade dos participantes emergiu dos seguintes critérios de inclusão: adolescentes com idades compreendidas entre os 10 e 19 anos de idade, a frequentar níveis de ensino entre o $5 .^{\circ}$ e o $12 .^{\circ}$ ano de escolaridade. Constituiram fatores de exclusão, os adolescentes com perturbaçóes músculo-esqueléticas secundárias, doenças do colagénio e infeciosas, onco-hematológicas, genéticas, endócrinas (como doenças da tiroide ou diabetes mellitus) e traumáticas recentes.

Como Instrumento de colheita de dados (ICD) foi utilizado um questionário, constituído por 4 secçôes: uma primeira com dados de cariz sociodemográfico;(Idade, género, zona de residência e classe socio-económica) uma segunda com dados antropométricos (peso, altura e IMC segundo a International Obesity Task Force), a terceira que procurava avaliar variáveis contextuais ( uso, caracteristicas e peso da mochila, tempo gasto por semana a ver televisáo, a jogar e a utilizar o computador, meio de transporte utilizado nas deslocaçóes para a Escola, práticas, tipologia e intensidade de atividade física); e a quarta e última secção integrava o Questionário Nórdico Músculo-esquelético validado e adaptado cultural e lingüísticamente para
Portugal, por Mesquita, Ribeiro \& Moreira em 2010. (10) O ICD integra vinte e cinco questôes de resposta dicotómica ( $\operatorname{sim} /$ não) e possui três perguntas correlacionando nove regióes anatómicas, identificadas com a ajuda de uma figura humana vista pela regiáo posterior. As questóes estão relacionadas com cada área anatómica, avaliando se o inquirido manifestou perturbações nos últimos 3 meses (opção para que as lembranças sejam mais recentes e com maior veracidade) e se ocorreram nesse período implicaçóes no seu dia-a-dia. Integra ainda, uma escala numérica da dor, para cada uma das áreas anatómicas, classificada num continuum entre 0 (sem dor) e 10 (dor máxima) para avaliar a dor. A colheita de dados foi efetuada nos meses de março, abril e maio de 2017, nos días em que decorriam as aulas de Educação Física.

Antes de se proceder à recolha dos dados foi solicitada aos pais dos adolescentes a colaboração voluntária e esclarecida no estudo com assinatura do consentimento informado. Foi fornecida informação acerca dos objetivos da investigação e assegurada a confidencialidade das respostas dadas, bem como dos juízos clínicos formulados, cumprindo os princípios éticos e legais. $\mathrm{O}$ estudo foi autorizado pela Comissão de Ética para a Saúde. Foi realizada análise estatística descritiva e inferencial com recurso ao Statistical Package for the Social Sciences (SPSS) versão 22.0. Para o estudo da relação entre variáveis foram utilizados testes paramétricos e não paramétricos, nomeadamente o Teste t de Student ou teste de U-Mann Whitney (UMW) para comparação de médias de uma variável quantitativa em dois grupos de sujeitos diferentes. Recorremos igualmente ao teste de Kruskall Wallis para comparação de médias de uma variável quantitativa em três ou mais grupos de sujeitos diferentes. Como complemento dado que se testou a igualdade de mais de duas médias, recorreu-se aos testes post hoc para determinar as que se diferenciam entre si. Nos testes estatísticos foi considerado um nível de significância de 5\%.

\section{Resultados}

Os adolescentes da amostra possuem idades que oscilam entre os 11 (Min) e 17 (Max) anos; com 
uma média de 13,36 anos e desvio padrão de 2,08, pertencendo $58,2 \%$ ao género feminino e $41,8 \%$ ao masculino. Habitam maioritariamente $(75,3 \%)$ em zonas rurais e pertencem a classes socioeconómicas intermédias (53,9\%).

Quanto ao estado ponderal verificámos que em média os adolescentes pesavam $53,89 \mathrm{Kg}$ $(\mathrm{D} p=11,44)$ mediam $1,59 \mathrm{~m}$ de altura $(\mathrm{Dp}=0,08)$ e a maioria $(71,5 \%)$ era eutrófica. Registar que $12,0 \%$ apresentava excesso de peso, estando este mais associado aos rapazes $\left(\mathrm{x}^{2}=6,341 ; \mathrm{p}=0,023\right)$, com diferenças estatísticas significativas.

A grande maioria dos adolescentes (97,5\%) transporta o material escolar em mochilas, que possuem alças ajustáveis (82,3\%) e acolchoadas $(78,5 \%)$ porém, sem cinto, correia de peito ou apoios metálicos. Observámos que 79,8\% se desloca para a escola de autocarro/carro, e apenas 20,2\% faz o percurso a pé ou de bicicleta. Transportam a mochila em média $20 \mathrm{mn} /$ dia, $80,0 \%$ nos dois ombros e 20,0\% (sobretudo raparigas) apenas num ombro. Constatámos ainda que para além da mochila, $87,3 \%$ dos participantes transporta outros equipamentos, essencialmente de apoio desportivo e educação visual e tecnológica. $\mathrm{O}$ peso relativo da mochila para a maioria dos participantes oscila entre $12 \%$ e $19 \%$ do seu peso corporal.

A maioria $(56,8 \%)$ dos adolescentes realiza atividade física regularmente, sobressaindo as modalidades futebol/futsal $(33,7 \%)$ e o basketball $(22,1 \%)$ com uma intensidade moderada. $72,8 \%$ dedica em média 2 horas dia a ver televisão, sendo as raparigas as maiores consumidoras, contudo as diferenças estatísticas não são significativas $(\mathrm{t}=1,087$; $\mathrm{p}=$ 0,342 ). A posição adotada por $38,6 \%$ dos inquiridos é a de semi-deitados no sofá. O tempo encontrado no uso das novas tecnologías (videos e computador) pelos adolescentes oscila entre $0 \mathrm{~h}$ e $32 \mathrm{~h}$ por semana, correspondendo-lhe uma média de 13,92 horas. Apesar dos valores médios diferirem entre rapazes e raparigas as diferenças estatísticas não são significativas $(\mathrm{p}=0.681)$.

A prevalencia das PME relatadas pelos adolescentes nos últimos 3 meses (Tabela 1) é de $(\mathrm{n}=300)$ $47,4 \%$, estando estas localizadas a vários níveis (respostas múltiplas) mas com maior enfoque no pescoço $(23,4 \%)$, ombros $(27,8 \%)$, região dorsal $(25,3 \%)$, regiáo lombar $(22,8 \%)$, .coxa/anca (26,1\%), e Joelhos (19,6\%).

Tabela 1. Prevalência das PME e regiões anatómicas afetadas

\begin{tabular}{lc}
\hline Perturbação musculo-esquelética & $\begin{array}{c}\text { Total } \\
\mathbf{n} \%\end{array}$ \\
\hline Sem perturbação & $332(52,6)$ \\
Com perturbação & $300(47,4)$ \\
Regiões corporais & \\
Pescoço & $70(23,4)$ \\
Ombros & $83(27,8)$ \\
Dorsal & $76(25,3)$ \\
Lombar & $68(22,8)$ \\
Coxa/Anca & $78(26,1)$ \\
Joelhos & $59(19,6)$ \\
\hline
\end{tabular}

A intensidade da dor referida pelos adolescentes com PME (Tabela 2) recai sobretudo nos itens dor fraca $(34,7 \%)$ e moderada $(41,3 \%)$, contudo observámos que $16,0 \%$ refere dor forte e $8,0 \%$ mesmo dor insuportável.

Tabela 2. Intensidade da dor sentida pelos adolescentes com PME

\begin{tabular}{lc}
\hline Intensidade da dor & Total \\
& $\mathbf{n}(\%)$ \\
\hline Dor fraca & $104(34,7)$ \\
Dor moderada & $124(41,3)$ \\
Dor forte & $48(16,0)$ \\
Dor insuportável & $24(8,0)$ \\
Total & $300(100)$ \\
\hline
\end{tabular}

Para analisar a relação existente entre variáveis independentes e as PME (variável dependente) nos adolescentes, utilizamos testes paramétricos e não paramétricos. Considerando o número elevado de variáveis, optámos por apresentar apenas aquelas que revelaram significância estatística. Deste modo verificámos que as PME são mais prevalentes nos adolescentes do género feminino $(\mathrm{t}=-3.358 ; \mathrm{p}=0,001)$ naqueles que gastam mais tempo por dia a ver televisão $\left(\chi^{2}=6,532 ; \mathrm{p}=0,038\right)$, e ainda naqueles que se deslocam para a escola a pé ou de bicicleta $(U=1531,500 ; p=0,018)$. Nas restantes variáveis (idade, área de residência, estado ponderal, transporte de mochila e pratica de exercício físico) não foram encontradas significâncias estatísticas $(\mathrm{p}>0,05)$.

\section{Discussão}

As características sociodemográficas dos adolescentes deste estudo estão alinhados com outros estudos 
realizados recentemente em contexto português e tendo por alvo esta mesma população. ${ }^{(7,11,12)}$ Tratase de uma amostra maioritariamente composta por adolescentes do género feminino, (58,2\%), com média de idade de13,36 anos, a residir em meio rural $(75,3 \%)$ e pertencentes a classes socioeconómicas intermédias $(53,9 \%)$, confirmando a correlação expectável com as condiçóes de vida das zonas do Interior do País. ${ }^{(13)}$

Do ponto de vista antroprométrico, pesavam em média 53,89 $\mathrm{Kg}$, mediam 1,59 m de altura e globalmente eram eutróficos (71,5\%). Não obstante, $12,0 \%$ apresentava excesso de peso, (sobretudo rapazes) o que favorece o aparecimento das PME como corroborado em outro estudo ${ }^{(13)}$ em que se afirma que os adolescentes com IMC acima do ideal apresentam frequentemente índices maiores de alteraçôes posturais de vários segmentos corporais. ${ }^{(14,15)}$

Vários autores chamam a atenção para o frequente uso inadequado das mochilas, por parte dos adolescentes, transportando cargas excesivas e de forma ineficiente, que os submete a incalculáveis e sérios desvios posturais. Como antídoto aconselham como limite seguro de carga nos adolescentes pesos que náo excedam $10 \%$ do seu peso corporal. $(16,17)$ De facto a maioria dos adolescentes $(97,5 \%)$ transporta o material escolar em mochilas com alças ajustáveis e acolchoadas, mas sem cinto, correia de peito ou apoios metálicos, o que contribui para uma maior tensão a nivel musculo-esquelético. Transportam a mochila em média $20 \mathrm{mn} /$ dia, e $20,0 \%$ deles (sobretudo raparigas) transporta a mochila apenas num ombro. Além disso, 87,3\% transporta outros equipamentos de apoio desportivo e de educação visual e tecnológica, o que concorre para o facto da maioria dos participantes transportar pesos que oscilam entre $12 \%$ e $19 \%$ do seu peso corporal. São dados que contrariam as recomendaçóes da OMS e da Direção Geral de Saúde Portuguesa, uma vez que estes organismos recomendam pesos que não excedam $10 \%$ do peso corporal como limite seguro. ${ }^{(16,18,19)}$

Ficou ainda demonstrado que a maioria (56,8\%) dos adolescentes estudados realiza atividade física regularmente (futebol/futsal e o basketball) com uma intensidade moderada. As evidências do impacto da atividade física sobre as PME nos adolescentes tem gerado muita polémica entre autores e estão longe de gerar consensos. São varios os investigadores que defendem que a falta de atividade física é responsável pelo surgimento de perturbações numa fase precoce da vida, porém o seu excesso é igualmente prejudicial, uma vez que levada a certos limites, (solicitando ao máximo músculos, tendôes, ossos e articulaçôes), a prática de atividade física pode atuar como agente patológico sobre o aparelho locomotor. ${ }^{(20,21)}$

A exposição dos adolescentes às tecnologias da informação tem aumentado no ambiente educacional, de lazer e familiar, sendo um fenómeno crescente no mundo e em todas as classes socioeconómicas. ${ }^{(3,8,22)}$ Pudemos observar que $72,8 \%$ dos nossos participantes dedica em média 2 horas dia a ver televisão, sendo a posição de eleição o estar deitado no sofá. O tempo médio encontrado no uso das novas tecnologías (videos e computador) é de 13,92 horas/semanas, exigindo posturas estáticas, repetidas e prolongadas com os danos consequentes. Concordamos que os benefícios do uso destas tecnologias são evidentes: maior acesso à informação de qualidade, possibilidade e troca de informação entre indivíduos e comunidades distantes, melhoria da habilidade cognitiva e até mesmo a disponibilização de atividades de lazer. Não obstante, o uso desregrado e abusivo é um fator que contribui para o sedentarismo e para alteraçóes posturais (com desajustes ergonómicos, proporçôes inadequadas do mobiliário na escola e em casa) e estruturais do esqueleto, com sobrecarga de articulaçôes, tendóes, ligamentos e músculos, provocando deformidades músculo-esqueléticas. ${ }^{(23,24)}$

Os resultados relativos à prevalencia, das PME dos adolescentes, não se podem dissociar dos aspetos referidos anteriormente e confirmam a tendência revelada por estudos epidemiológicos de ser elevada. ${ }^{(3,8,22)} \mathrm{Na}$ verdade $47,4 \%$ dos nossos participantes apresenta PME localizadas em zonas corporais diversas, (e simultâneas). Um estudo nacional $^{(12)}$ sobre a saúde dos adolescentes demonstrou que $35 \%$ dos adolescentes referem ter dor ao nível do pescoço/ombro e $38,6 \%$ refere dor a nível lombar. Os resultados de outros investigado- 
res identificam cenários com características muito semelhantes aos do nosso estudo. ${ }^{(3,7,8,12)}$

A dor sentida nos segmentos corporais apontados é para a maioria dos adolescentes de intensidade moderada $(41,3 \%)$, e fraca $(34,7 \%)$, contudo não deve ser desvalorizado o facto de $16,0 \%$ referir dor forte e $8,0 \%$ mesmo dor insuportável. A International Association for the Study of Pain (IASP) define a dor como uma experiencia desagradável sensorial ou emocional, associada a um potencial ou real dano tecidual, e quando associada à população adolescente, não afeta apenas o estado de saúde atual, mas predispóe ao desenvolvimento da dor crónica na idade adulta, com profundas implicaçóes na sua qualidade de vida. ${ }^{(25,26)}$ De facto a dor não pode ser entendida como uma manifestaçáo isolada e ocasional, mas sim como uma ocorrência com consequências por vezes dramáticas no desenvolvimento da saúde e bem estar do ser humano ao longo da vida e na sustentabilidade dos próprios sistemas de saúde dos Países.

A análise da relação entre variáveis revelou que as PME são mais prevalentes nos adolescentes do género feminino. Esta diferença entre géneros pode ser explicada tendo em conta um diferente limiar na perceção da sintomatologia músculo-esquelética, uma maior flexibilidade muscular no género feminino, ou ain$\mathrm{da}$ alteraçôes hormonais durante a puberdade. ${ }^{(27)} \mathrm{Os}$ participantes que gastam mais tempo por dia a ver televisão e usam por períodos mais longos as novas tecnologias apresentam também uma maior prevalência de PME. São dados que reforçam os de outros investigadores, ao afirmar que o uso frequente das tecnologias informáticas constitui um fator de risco elevado ao desenvolvimento das PME. ${ }^{(23,24)}$ Similarmente as deslocaçóes para a escola a pé ou bicicleta constitui um fator de risco à instalação das perturbaçóes, dado que a carga da mochila associada ao equilíbrio em movimento provoca deslocamentos posteriores do centro de gravidade do corpo e este desequilíbrio é compensado com a projeção do corpo para a frente, podendo levar ao desvio da coluna. ${ }^{(12,14,28)}$ Os resultados deste estudo direcionam para a necessidade de um maior investimento na prevenção destas perturbaçóes na adolescência, como um dos pilares do Serviço Nacional de Saúde, numa lógica de promoção e continuidade de cuidados, podendo ser um recurso muito vantajoso para os indivíduos, para as famílias e para a sociedade em geral.

Como limitaçóes do estudo, apontam-se: a utilização de uma amostra do tipo não probabilístico por conveniencia, o que não nos permite generalizar resultados com precisão estatística; recolha de dados efetuada por auto preenchimento do questionário pelos adolescentes, o que pode apresentar alguns vieses de interpretação quer por substimação ou hipervalorização do problema das PME e deste modo, afetar a validade dos dados; integração de questóes simples na avaliação da prática de atividade física em vez de uma escala para avaliação da atividade física e sedentarismo, de que é exemplo a "WHO HBSC - World Health Organisation health bahavior in schoolchildren", que nos permitiria classificar os adolescentes em "ativos", "inadequadamente ativos" e "inativos", o que inviabilizou conhecer com maior precisão e confiabilidade os dados relativos a esta variável.

Pese embora as limitaçóes referidas, consideramos que o estudo aborda uma temática muito pertinente na atualidade, aportando os seus resultados contributos interessantes ao conhecimento científco em Enfermagem. Como sugestão propomos, o desenvolvimento de futuros estudos, comparando no mesmo período de tempo e numa perspetiva longitudinal, a evoluçáo do potencial de saúde das pessoas ao longo do ciclo vital.

\section{Conclusão}

Os adolescentes que constituíram a amostra deste estudo apresentam, como pudemos constatar, uma prevalência elevada de PME. O desconforto referido, localiza-se fundamentalmente nas regióes anatómicas do pescoço, ombros, zona dorsal, zona lombar, coxas/ancas, e Joelhos. Apesar do padrão de intensidade da dor se ter revelado para a maioria dos adolescentes entre o moderado e fraco, há que considerar avaliaçóes distintas e com gravidade crescente ao ser referida dor forte e mesmo insuportável. Como era expectável face aos determinantes referenciados em estudos similares, as PME deste grupo de adolescentes são mais prevalentes nas raparigas, naqueles que gastam mais tempo por dia no uso de novas tecnolo- 
gias e ainda no tipo de transporte utilizado nas deslocaçóes para a escola, uma vez que a deslocação a pé ou de bicicleta se tornam mais penosas. Em síntese final, podemos afirmar que as perturbaçóes músculo-esqueléticas nos adolescentes, têm uma origem dinâmica, multifacetada e multidimensional pois se por um lado existem fatores que assumem particular importância por concorrerem diretamente para a ocorrência destas manifestaçóes (como os de origem mecânica), outros influenciam indiretamente, sobretudo os de origem social, cultural e organizacional.

\section{Agradecimentos}

Os nossos agradecimentos ao Fundo Social Europeu, ao Programa Operacional CENTRO 2020 e à Fundação para a Ciência e Tecnologia do Governo Português, instituiçóes públicas que se constituem como fontes de financiamento do Projeto de Investigação MAISaúde Mental "Monitorização e avaliação dos indicadores de saúde mental das crianças e adolescentes: Da investigação à prática” (código de identificação:CENTRO-01-0145-FEDER-023293), onde este estudo se integra.

\section{Colaborações}

Martins RL, Carvalho N, Albuquerque C, Andrade A, Martins C, Campos S, Batista S e Dinis AI contribuíram com a concepção do estudo, análise e interpretação dos dados, redação do artigo, revisão crítica relevante do conteúdo intelectual e aprovação da versão final a ser publicada.

\section{Referencias}

1. World Health Organization. Evaluation of the national health plan of Portugal: 2004-2010. Geneva: WHO; 2010.

2. Kamper SJ, Henschke N, Hestbaek L, Dunn KM, Williams CM. Musculoskeletal pain in adolescence. Braz J Phys Ther. 2016;16;20(3):275-84.

3. Martins R, Almeida A, Andrade Moreira H, Campos S. Prevalência e determinantes das perturbações músculo-esqueléticas em adolescentes. Psicol Criança Adolesc. 2016;7(1-2):73-82.
4. Meziat Filho N, Coutinho ES, Azevedo e Silva G. Association between home posture habits and low back pain in high school adolescents. Eur Spine J. 2015;24(3):425-33.

5. Martins R, Albuquerque C, Andrade A, Martins C, Campos S. Subjective health associated with the quality of life of adolescents. In: 4th International Conference on Health and Health Psychology. The European Proceedings of Social \& Behavioural Sciences; 2018 Jul 4-6; Viseu: Escola Superior de Saúde de Viseu; 2018. p. 149-57.

6. Pinho ME, Vaz MA, Arezes PM, Campos JC, Magalhães AP. Lesões músculo-esqueléticas relacionadas com as atividades desportivas em crianças e adolescentes: uma revisão das questões emergentes. Motricidade. 2013;9(1):31-49.

7. Fonseca CD, Candotti CT, Noll M, Luz AM, Santos AC, Corso CO. Prevalence of back pain among high school students in a municipality in southern Brazil. Fisioter Mov. 2016;29(1):137-46.

8. Albuquerque C, Santos C, Martins R, Cunha M. Strategies for the prevention of work-related musculoskeletal injuries: Systematic review of literature. In: 3rd International Conference on Health and Health Psychology, The European Proceedings of Social \& Behavioural Sciences; 2017 jul 5-7. Porto: Faculdade de Medicina, Universidade do Porto. p. 79-89

9. Noll M, Candotti CT, Rosa BN, Loss JF. Back pain prevalence and associated factors in children and adolescents: an epidemiological population study. Rev Saude Publica. 2016;50. pii: S0034-89102016000100219.

10. Mesquita CC, Ribeiro JC, Moreira P. Portuguese version of the Standardize Musculoskeletal Questionnaire: cross cultural and reability. J Public Health. 2010;18(5):461-6.

11. Trigueiro MJ, Massada L, Garganta R. Back pain in Portuguese school children: prevalence and risk factors. Eur J Public Health. 2013 Jun;23(3):499-503.

12. Health Behaviour in School. A saúde dos adolescentes portugueses em tempos de recessão: Dados nacionais do estudo HBSC de 2014 [Internet]. Lisboa: Aventura Social \& Saúde; 2014. [citado 2019 Jul 21]. Disponível em: http://aventurasocial.com/arquivo/1437158618_ RELATORI0\%20HBSC\%202014e.pdf

13. Instituto Nacional de Estatística (INE). Crianças e Adolescentes em Portugal. Rev Estud Demográficos; 2015;55:53-101.

14. Sedrez JA, da Rosa MI, Noll M, Medeiros FS, Candotti CT. Fatores de risco associados a alterações posturais estruturais da coluna vertebral em crianças e adolescentes. Rev Paul Pediatr. 2015;33(1):72-81.

15. Tsekoura M, Dimitriadis Z. Kastrinis Al, Katsoulaki M, Nomikou El. Association of musculoskeletal pain with schoolbags. Interscientific Health Care. 2017;9(3):86-91.

16. World Health Organization (WHO). Global recommendations on physical activity for health. Geneva: WHO; 2016.

17. Kunzler M, Noll M,Antoniolli A, Candotti CT. Associação entre postura sentada e alterações posturais da coluna vertebral no plano sagital de escolares da cidade de Lajeado, RS. Rev Baiana Saúde Pública. 2014;38(1):197-212.

18. Portugal. Ministério da Saúde Direção Geral de Saúde. Plano Nacional de Saúde Escolar [Internet]. Lisboa: DGS; 2015. [citado 2019 Jul 21]. Disponível em: http://pns.dgs.pt

19. Natasha AA, Syukria AA, Kamaruddin M, Diana SN. Ima-NirwanaS, Chin KY. The association between backpack use and low back pain among pre-university students: A pilot study. J Taibah University Med Sci. 2018;13(2):205-9.

20. Calvo-Muñoz I, Gómez-Conesa A, Sánchez-Meca J. Physical therapy treatments for low back pain in children and adolescents: a metaanalysis [Internet]. BMC Musculoskelet Disord. 2013;14(1):55. 
21. O'Keeffe M, O'Sullivan K, Kamper SJ. Schoolbags and back pain: opinions strongest where the evidence is weakest. Physiotherapy. 2018;104(1):e1.

22. Minghelli B, Oliveira R, Nunes C. Postural habits and weight of backpacks of Portuguese adolescents: are they associated with scoliosis and low back pain? Work. 2016;54(1):197-208.

23. Yamato TP, Maher CG, Traeger AC, Wiliams CM, Kamper SJ. Do schoolbags cause back pain in children and adolescents? A systematic review. Br J Sports Med. 2018;52(19):1241-5.

24. Batista IT, Melo-Marins D, Carvalho RG, Gomes LE. Weight and mode of carrying schoolbags at elementary school: effect of the school grades and sex. Fisioter Pesqui. 2016;23(2):210-5.
25. Bilik 0, Karayurt 0, Savci A, Turhan Damar A. Experiências de adolescentes e suas famílias a curto prazo após cirurgia para correção de escoliose. Acta Paul Enferm. 2018;31(4):342-50.

26. International Association for the Study of Pain. Classification of chronic pain. Seattle, Washington: IASP Press; 2002.

27. Manchikanti L, Hirsch JA. What can be done about the increasing prevalence of low back pain and associated comorbid factors? Pain Manag. 2015;5(3):149-52.

28. Tsekoura M, Dimitriadis Z. Kastrinis Al, Katsoulaki M, Nomikou El. Association of musculoskeletal pain with schoolbags. Interscientific Health Care. 2017;9(3):86-91. 